\title{
Android Image Classifier
}

\author{
Piyush Bansal $^{1}$ | Saurabh Gautam ${ }^{1}$ \\ Department of Information Technology, Maharaja Agrasen Institute of Technology, Delhi, India
}

\section{To Cite this Article}

Piyush Bansal and Saurabh Gautam, "Android Image Classifier", International Journal for Modern Trends in Science and Technology, 6(12): 275-276, 2020.

\section{Article Info}

Received on 10-November-2020, Revised on 02-December-2020, Accepted on 06-December-2020, Published on 11-December-2020.

\section{ABSTRACT}

Image classification is the task of identifying an image. Android image classification model is trained to recognize various classes of images. For example, we may train a model to recognize photos representing three different types of animals: rabbits, hamsters, and dogs. Optimized pre-trained models are provided byTensor Flow Lite that we can deploy in our mobile applications. Simple Machine Learning (ML) algorithms in Python make relatively easy to start explore datasets and make some first predictions. We can make these trained models useful in the real world by making them available to make predictions on either the Web or Portable devices.

KEYWORDS: Image classification, Tensor Flow Lite

\section{INTRODUCTION}

With the use of computer Lillsand and Kiefer defined image processing as involving manipulation of digital images. It is a broad subject and involves processes that are mathematically complex. Image processing involves some basic operations like image enhancement, image classification, etc. Image classification is a very important part of image processing. The objective of image classification is that the automatic allocation of image to thematic classes. Two types of classification are supervised classification and unsupervised classification

Deep structured learning, or more commonly called deep learning has emerged as a new area of machine learning research since 2006. Several definitions are available for Deep Learning; coating one of the many definitions from. Deep Learning is defined as: A class of machine learning techniques that exploit many layers of nonlinear information processing for supervised or unsupervised feature extraction and transformation and for pattern analysis and classification.

\section{RESULTS}

After applying various Machine Learning Models on our dataset we found out that Logistic Regression Model was able to give us the best result. Here our primary goal was to arrive or rather select a model which would be able to provide us with least amount of false negative. On using various models we found that Logistic regression was able to provide us with a specificity of $90.9 \%$ with only $3 \%$ of the results being classified as false negative.

The various models result accuracy with confusion matrix for the given dataset were as follows. 


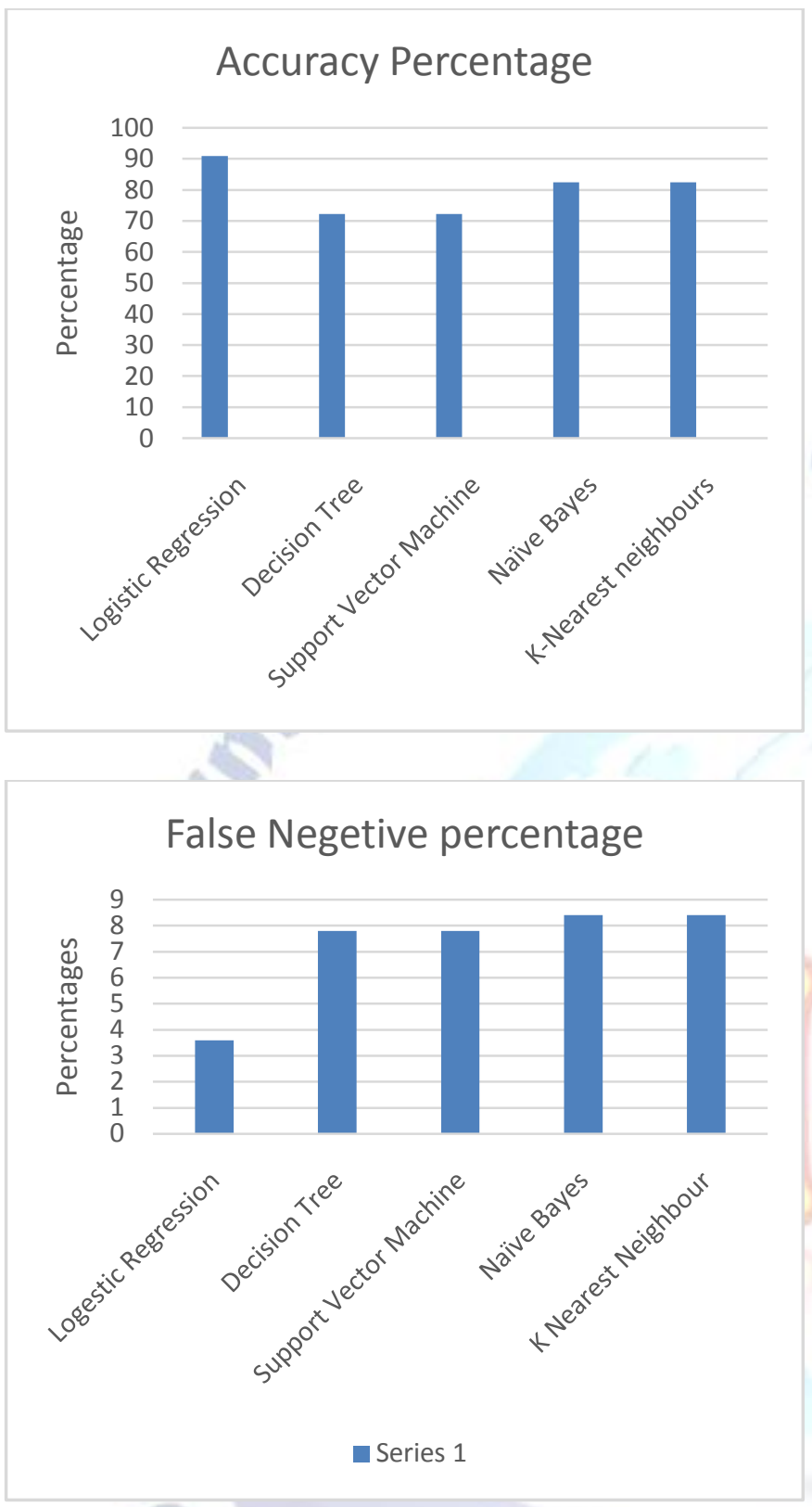

\section{DISCUSSION}

It is clear that the use of Machine Learning very useful when one is tasked with producing accurate decisions based on complicated data sets. But they come with some significant challenges and limitations that you either have to accept or try to overcome. When deploying any machine learning model, one is instantly faced with challenges related to data access, privacy issues, data protection, and more. And when the data is someone's personal data like here the question of security becomes really important.

In future I would likely to add few more features like image enhancing, text translation, etc. Image enhancing includes the camera apps in smart phones and digital cameras use image processing to enhance the image quality, video stabilization and noise removal etc. Text translation means to translate the text in the image to desired language. Moreover I would also connect my app to websites like Wikipedia so that the information on the items can be available. I may also use multiple input modes to give video and photo inputs.

\section{IV.REFERENCES}

[1] https://www.python.org/doc/

[2] https://developer.android.com/guide?authuser=2

[3] http://www.image-net.org/

[4] https://arxiv.org/abs/1704.04861

[5] https://github.com/alseambusher/Paideia

[6] https://www.tandfonline.com/doi/full/10.1080/0143116 0600746456

[7] https://ieeexplore.ieee.org/abstract/document/8340197 